\title{
Cross-Talk between Human Dendritic Cell Subsets Influences Expression of RNA Sensors and Inhibits Picornavirus Infection
}

\author{
Matthijs Kramer $^{a} \quad$ Barbara M. Schulte ${ }^{b}$ Dagmar Eleveld-Trancikova ${ }^{a}$ \\ Maaike van Hout-Kuijer ${ }^{a} \quad$ Liza W.J. Toonen $^{a} \quad$ Jurjen Tel ${ }^{\mathrm{a}} \quad$ I. Jolanda M. de Vries ${ }^{\mathrm{a}}$ \\ Frank J.M. van Kuppeveld ${ }^{b}$ Bastiaan J.H. Jansen ${ }^{a}$ Gosse J. Adema ${ }^{a}$ \\ Departments of a Tumor Immunology and ${ }^{\mathrm{b}}$ Medical Microbiology, Nijmegen Centre for Molecular Life Sciences, \\ Radboud University Nijmegen Medical Centre, Nijmegen, The Netherlands
}

\section{Key Words}

Human · Dendritic cells · Cell activation • RNA sensor •

Cross-talk

\begin{abstract}
Dendritic cells (DCs) are professional antigen-presenting cells that provide a link between innate and adaptive immunity. Multiple DC subsets exist and their activation by microorganisms occurs through binding of conserved pathogenderived structures to so-called pattern recognition receptors (PRRs). In this study we analyzed the expression of PRRs responding to viral RNA in human monocyte-derived DCs (moDCs) under steady-state or pro-inflammatory conditions. We found that mRNA and protein levels for most PRRs were increased under pro-inflammatory conditions, with the most pronounced increases in the RIG-like helicase (RLH) family. Additionally, freshly isolated human plasmacytoid DCs (pDCs) displayed significantly higher levels of TLR7, RIGI, MDA5 and PKR as compared to myeloid DCs and moDCs. Finally, we demonstrate for the first time that cross-talk between TLR-matured or virus-stimulated pDCs and moDCs leads to a type I interferon-dependent antiviral state in moDCs. This antiviral state was characterized by enhanced $\mathrm{RLH}$ expression and protection against picornavirus infec-
\end{abstract}

tion. These findings might represent a novel mechanism by which pDCs can preserve the function and viability of myeloid DCs that are attracted to a site with ongoing infection, thereby optimizing the antiviral immune response.

Copyright $\odot 2010$ S. Karger AG, Basel

\section{Introduction}

Dendritic cells (DCs) are specialized antigen-presenting cells that are crucial players in the decision process between tolerance and immunity. They participate in the innate immune response, but also orchestrate adaptive immunity via regulation of $T$ cell activation and differentiation $[1,2]$. Different DC subsets exist that are specialized in regulation of distinct facets of the immune response. For instance, plasmacytoid DCs (pDCs) are well equipped to recognize viral structures, resulting in potent type I interferon (IFN) production [3]. Myeloid DCs (mDCs) can recognize structures derived from several classes of microorganisms and are key to the induction of Th1 responses via release of IL-12 [3-5]. For in vitro stud-

\section{M.K. and B.M.S. contributed equally.}

\section{KARGER}

Fax +41613061234 E-Mail karger@karger.ch www.karger.com

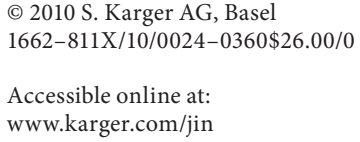

Department of Tumor Immunology, Nijmegen Centre for Molecular Life Sciences Radboud University Nijmegen Medical Centre

Geert Grooteplein 28, NL-6525 GA Nijmegen (The Netherlands)

Tel. +31 24361 7600, Fax +31 24354 0339, E-Mail g.adema@ncmls.ru.nl 
ies, monocyte-derived DCs (moDCs) are often used instead of mDCs. Responses of moDCs are similar to mDCs in many aspects $[3,6]$, yet differences exist $[7,8]$.

Recognition of microorganisms occurs via binding of pathogen-associated molecular patterns (PAMPs) to socalled pattern-recognition receptors (PRRs), such as the cytoplasmic NOD-like receptors and RIG-I-like helicases and the membrane-bound C-type lectins and Toll-like receptors (TLRs) [9]. Triggering of TLRs on DCs by PAMPs, such as double-stranded RNA (dsRNA) or lipopolysaccharide (LPS), results in production of pro- and anti-inflammatory cytokines and upregulation of MHC and costimulatory molecules that collectively determine $\mathrm{T}$ cell activation and differentiation [10]. Despite their crucial role in antiviral immunity, TLRs that recognize viral RNA are unable to detect viruses in the cytoplasm, due to their endosomal localization. However, the recently identified PRR family called the RIG-I-like helicases (RLHs) appears to be involved in responses to cytoplasmic viral RNA. The RLHs retinoic acid-inducible gene I (RIG-I) and melanoma differentiation-associated gene 5 (MDA5) respond to different RNA viruses through recognition of distinct RNA structures [11-14]. Activation of RIG-I or MDA5 is of crucial importance for the induction of innate antiviral immune responses upon virus infection [15]. The third member of the RLH family, LGP2, lacks a signaling domain and is thought to serve as a negative regulator [16]. Another RNA sensor that does not belong to the RLH family is protein kinase R (PKR), which inhibits translation upon detection of dsRNA in the cytoplasm [17].

Unlike TLRs, much less is known regarding the function and expression of RLHs. The vast majority of studies concerning RLHs have been done in mice or cell lines, with little data on expression of these molecules in primary human cells. In this study, we investigated the expression levels of TLRs and RLHs in human moDCs under steady-state or pro-inflammatory conditions. In addition, we determined the expression profiles of RNA sensors in different primary human DC subsets which revealed remarkably high expression of RLHs in pDCs compared to their myeloid counterpart. Differential expression of PRRs likely tailors the response to specific pathogens executed by various DC subsets. Cross-talk between DCs subsets might further aid in induction of optimal immune responses. We therefore determined the influence of cross-talk between pDCs and moDCs, which showed that TLR- or virus-activated pDCs increased expression of RLHs in moDCs and induced protection against picornavirus infection.

\section{Materials and Methods}

Culture and Stimulation of Cells

moDCs were generated from different donors as described previously [18]. Immature moDCs were harvested on day 6 using cold PBS and incubated with medium (RPMI-1640) alone or stimulated with purified LPS $(100 \mathrm{ng} / \mathrm{ml})+\operatorname{IFN}-\gamma(400 \mathrm{U} / \mathrm{ml})$ at $37^{\circ} \mathrm{C}$ for a period of 4,8 or $24 \mathrm{~h}$. Alternatively, moDCs were stimulated using $100 \mathrm{U} / \mathrm{ml}$ IFN $\alpha 2$ (Roferon- ${ }^{\circledR}$; Roche) or a 1:100 dilution of cell-free supernatant of CpG-C or R848-activated pDCs (collected $24 \mathrm{~h}$ after stimulation) or virus-simulated pDCs (see below). Myeloid and pDCs were isolated using anti-BDCA-1 and antiBDCA-4-conjugated magnetic microbeads, respectively, according to the manufacturer's instructions (Miltenyi Biotec).

\section{RNA Isolation}

Total RNA was isolated from DC cultures using TRIZOL reagent (Invitrogen Life Technologies) according to the manufacturer's instructions. RNA integrity was determined by analyzing the ribosomal $28 \mathrm{~S}$ and $18 \mathrm{~S}$ bands on a $1 \%$ agarose gel. The reverse transcription reaction was performed using Moloney murine leukemia virus reverse transcriptase (Invitrogen) according to the manufacturers' instructions. To exclude genomic DNA contamination we included a '-RT' control for each sample in which the reverse transcriptase was replaced by DEPC-treated milli- $\mathrm{Q}$ after which quantitative PCR (qPCR) on a single-exon gene (SOCS-1) was performed. The CDNA was stored at $-20^{\circ} \mathrm{C}$ until further use.

\section{Quantitative PCR}

qPCR analysis of gene expression in DCs was performed using TaqMan ${ }^{\circledR}$ Custom Arrays (Applied Biosystems) based on microfluidic card technology. Arrays were run on the ABI PRISM 7900 HT Sequence Detection System and data were analyzed using SDS 2.2.2 software (Applied Biosystems). Alternatively, qPCR data were obtained using SYBR Green (Applied Biosystems) based qPCR according to the manufacturers' instructions. Primer sequences are available upon request and were from the Primer Bank database [19]. Reactions were performed on an ABI PRISM 7900 Sequence Detection System (Applied Biosystems). Data were analyzed using 7000 System SDS software (Applied Biosystems). Genes with a $\mathrm{Ct}$ value $>37$ were considered not expressed. $\Delta \mathrm{Ct}$ values were calculated for all individual genes in all donors, with that of porphobilinogen deaminase (PBGD, also known as hydroxymethylbilane synthase) as a reference. An Excel macro was used to generate a heat map, where 1 gray scale encompasses $2 \Delta \mathrm{Ct}$ units. White indicates highest expression, whereas black corresponds to undetectable expression.

\section{Confocal Microscopy}

moDCs or pDCs were harvested, washed and allowed to adhere to poly-L-lysine coated coverslips in serum-free medium for $1 \mathrm{~h}$ at $37^{\circ} \mathrm{C}$. Cells were fixed with $1 \%$ paraformaldehyde and blocked in PBS with 3\% BSA, 10 mM glycine and 2\% human serum (blocking buffer, BB). For cell surface stainings, moDCs and pDCs were incubated using mouse-anti-human DC-SIGN (Beckman Coulter) or mouse-anti human BDCA4 (Miltenyi Biotec $\mathrm{GmbH}$ ), respectively, or the appropriate isotype control in BB. Following incubation and washes, cells were incubated with isotype-specific Alexa-labeled goat-anti-mouse IgGs (Molecular Probes). For intracellular staining, cells were fixed using $1 \%$ para- 
formaldehyde, permeabilized using $0.1 \%$ Triton-X100 in PBS and incubated with rabbit polyclonal anti-MDA5 [20] followed by incubation with goat-anti-rabbit IgG Alexa 488 (Pharmingen) in BB. Cells were sealed using Mowiol (Merck) and analyzed by confocal laser scanning microscopy on an MRC1024 confocal microscope (BioRad). Signals were collected sequentially to avoid bleed through and were processed with Photoshop 7.0 software (Adobe Systems Inc.).

\section{Western Blot}

Equal amounts of protein were separated by 7.5\% SDS-PAGE, electroblotted onto nitrocellulose membranes (Bio-Rad), followed by probing with the indicated antibodies. Anti-RIG-I and anti-PKR antibodies were purchased from ProSci Incorporated and Becton Dickinson Transduction Laboratories, respectively. RIG-I, PKR and MDA5 antibodies were used in 1:1,000, 1:500 and 1:10,000 dilutions, respectively. After washes, membranes were incubated with IRDye anti-mouse or anti-rabbit IgG $(1: 15,000$; Li-Cor Biosciences). Imaging was done using the Odyssey System.

Virus Stimulation of $p D C s$ and Infection of moDCs

Echovirus 9 Hill (EV9) and Coxsackievirus B3 Nancy (CVB3) were propagated as described before [18]. pDCs, isolated as described above, were stimulated with CVB3 at a multiplicity of infection (MOI) of 100 in the presence or absence of $10 \%$ human serum. After $24 \mathrm{~h}$, supernatants are harvested and used in a 1:10 dilution to stimulate moDC cultures. Unstimulated and stimulated moDCs were harvested using cold PBS, washed and infected with EV9 at an MOI of 1 in serum-free RPMI. After a 60-min incubation at $37^{\circ} \mathrm{C}$, cells were washed 3 times in an excess volume of PBS after which viral titers were determined at different time points post infection as described before [18]. To exclude CVB3 interference during EV9 replication analysis, titrations were done in the presence of neutralizing anti-CVB3 antibodies.

\section{Statistical Analysis}

Differential expression was assessed by means of one-factor ANOVA, and nonparametric pairwise comparison of the DC subsets was done using the Tukey's post hoc test in the R statistics package [21]. A p value of $<0.05$ was considered a significant difference.

\section{Results}

\section{TLR and RLH Profiles in Human Immature and} Mature Monocyte-Derived DCs

mRNA levels of TLRs, RLHs and other PRRs in human moDCs from three different donors were determined using custom-designed low-density arrays based on microfluidic card technology (see Materials and Methods) and qPCR. In line with their function as 'sentinels' of the body, human immature DCs were found to express a broad variety of PRRs. Regarding TLRs responding to RNA, that is TLR3, TLR7 and TLR8, moderate steady-state levels were observed (fig. 1a). In con- trast to their RNA-sensing TLR counterparts, significant transcript levels of the RLHs RIG-I, MDA5 and LGP2 were detected in all donors, along with high levels of the dsRNA activated anti-viral effector molecule PKR. The levels of RIG-I and MDA5 were as much as 25to 100 -fold higher compared to expression of TLR3 or TLR7, implying an important role for RLHs in moDC biology (fig. 1a). Furthermore, transcripts for essentially all TLRs were detected, as well as a broad panel of C-type lectin receptors (online suppl. fig. 1, www. karger.com/doi/10.1159/000300568) that can bind many pathogens, among which different viruses, and potentially function as entry receptors $[22,23]$.

To study potential changes in PRR mRNA levels under pro-inflammatory conditions, we stimulated DCs with LPS $(100 \mathrm{ng} / \mathrm{ml})+\mathrm{IFN}-\gamma(400 \mathrm{U} / \mathrm{ml})$ for $24 \mathrm{~h}$. Some members of the TLR family showed a modest up- or downregulation following exposure to LPS/IFN- $\gamma$, and significant donor differences were observed. Also the expression of C-type lectin receptors showed moderate changes (online suppl. fig. 1). Among the TLRs, the largest increases were found in the RNA-sensing TLRs (fig. 1a; online suppl. fig. 1). TLRs involved in the detection of bacteria, such as TLR1, TLR2 and TLR4, whose expression levels were already substantial, showed only a modest increase. Interestingly, differences in expression levels were more apparent within the family of cytoplasmic viral RNA sensors. Expression of RLHs RIG-I and MDA5 as well as effector molecules like PKR were significantly upregulated following stimulation with LPS/IFN- $\gamma$, suggesting that exposure to these pro-inflammatory stimuli can affect the responsiveness of DCs to viral pathogens.

\section{Dynamics of PRR Expression upon DC Stimulation}

Next, we analyzed kinetics of PRR expression following stimulation of DCs, focusing on PRRs involved in recognition of viral RNA, that is TLR3, TLR7, TLR8 and the cytoplasmic viral RNA sensors. Upregulation of TLRs and RLHs peaked approximately $8 \mathrm{~h}$ after exposure to pro-inflammatory stimuli, and expression of most genes had returned to baseline levels after $24 \mathrm{~h}$ (fig. 1b). TLR3 expression showed surprisingly little modulation in time compared to the other TLRs and RLHs studied (fig. 1b, right panel), whereas RIG-I and MDA5 mRNA expression increased more than 50-fold (fig. 1b, left panel). Western blot analysis corroborated our PCR results on protein level by showing enhanced expression of RIG-I, MDA5 and PKR following $24 \mathrm{~h}$ treatment with LPS/ IFN- $\gamma$ (fig. 1c). Thus, upon exposure to PAMPs and proinflammatory stimuli, moDCs rapidly upregulate ex- 


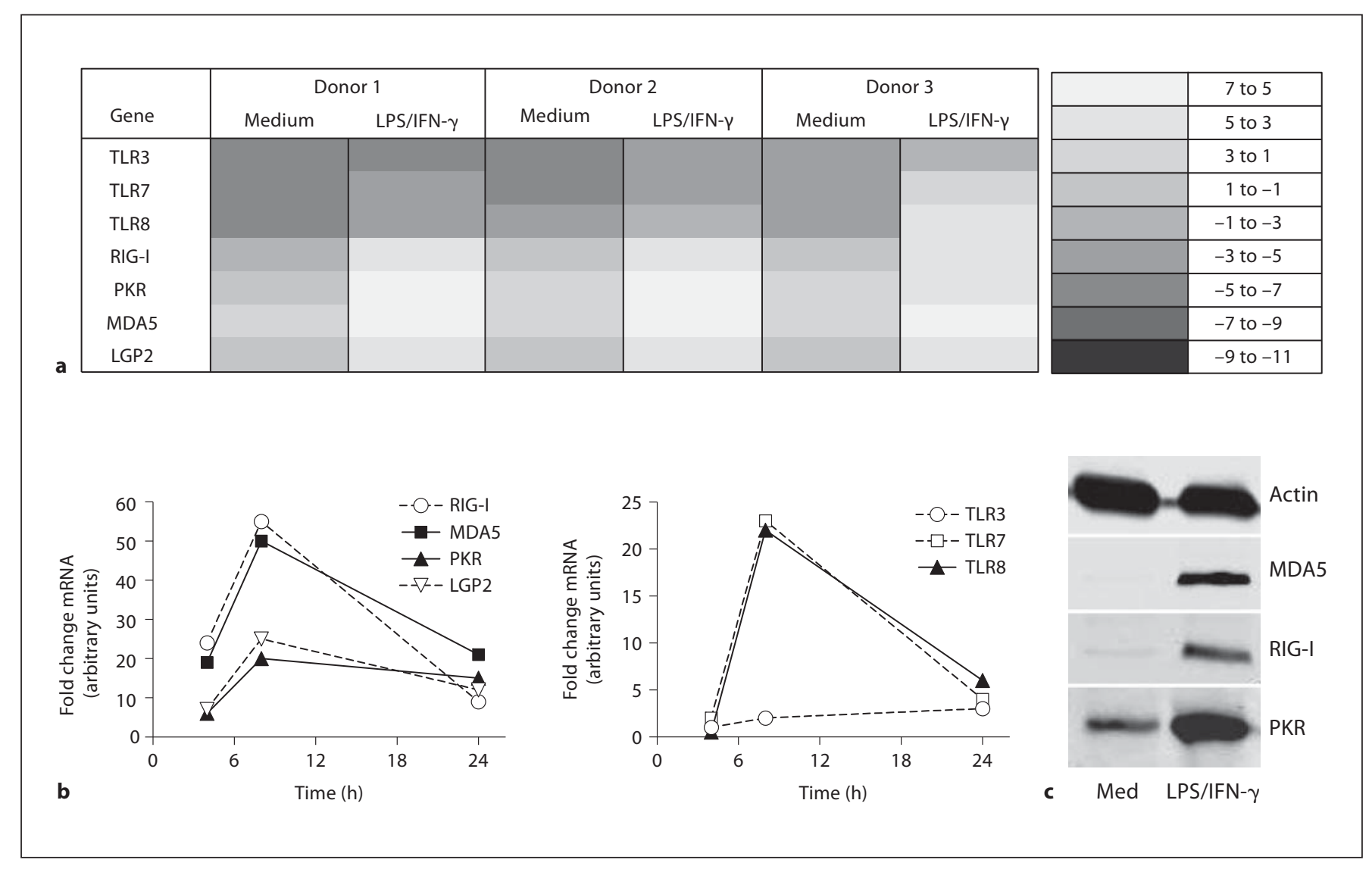

Fig. 1. Expression of RNA sensors in human moDCs in steadystate and under pro-inflammatory conditions. a Human moDCs were obtained by culturing freshly isolated blood monocytes from 3 different donors with IL-4 and GM-CSF. Unstimulated moDCs or DCs stimulated with LPS $(100 \mathrm{ng} / \mathrm{ml})+\mathrm{IFN}-\gamma(400 \mathrm{U} / \mathrm{ml})$ for $24 \mathrm{~h}$ were used. Total RNA of unstimulated, or stimulated cells was isolated and quantitative mRNA expression levels of the indicated genes were analyzed using low-density arrays and conventional qPCR. $\Delta \mathrm{Ct}$ values were calculated with the $\mathrm{Ct}$ value for PBGD as a reference. One gray-scale color encompasses a 2- $\Delta \mathrm{Ct}$ range. Black corresponds to undetectable mRNAs. b Immature
moDCs of 3 different donors were stimulated as described for a and total RNA was isolated 4, 8 or $24 \mathrm{~h}$ after stimulation. Quantitative mRNA expression levels of the indicated genes were analyzed using conventional qPCR and are displayed relative to unstimulated cells (put to 1). PBGD was used as reference gene. One representative example of 3 independent experiments using different donors is shown. c DCs were stimulated as described for a, and protein levels of RIG-I, MDA5, PKR and actin were analyzed using Western blot $24 \mathrm{~h}$ after stimulation. Shown is a representative example from 2 independent experiments using different donors. pression of PRRs responding to viral RNA at the mRNA and protein level, which might result in an increased capacity to respond to microorganisms.

\section{Expression of RNA Sensors in Blood Myeloid DCs and} Plasmacytoid DCs, and moDCs

To further study the RLH-family of RNA sensors in human DC subsets, we determined their expression profile in moDC, freshly isolated mDCs and plasmacytoid (p)DCs, which play a crucial role in antiviral immunity. The expression of TLR3, TLR7 and TLR8 that are also involved in viral RNA recognition was analyzed in parallel. Quantitative analysis showed that TLR8 levels were approximately 10 -fold higher than levels of TLR3 or TLR7 in moDCs, while differences in expression of these TLRs in freshly isolated mDCs were less pronounced (fig. 2a). In contrast, pDCs were found to express approximately 100-fold higher levels of TLR7 as compared to TLR8 and as much as 500 times more TLR7 than TLR3. In addition, highly divergent TLR expression profiles were detected between the different DC subsets analyzed. The most pronounced TLR3 expression was found in 


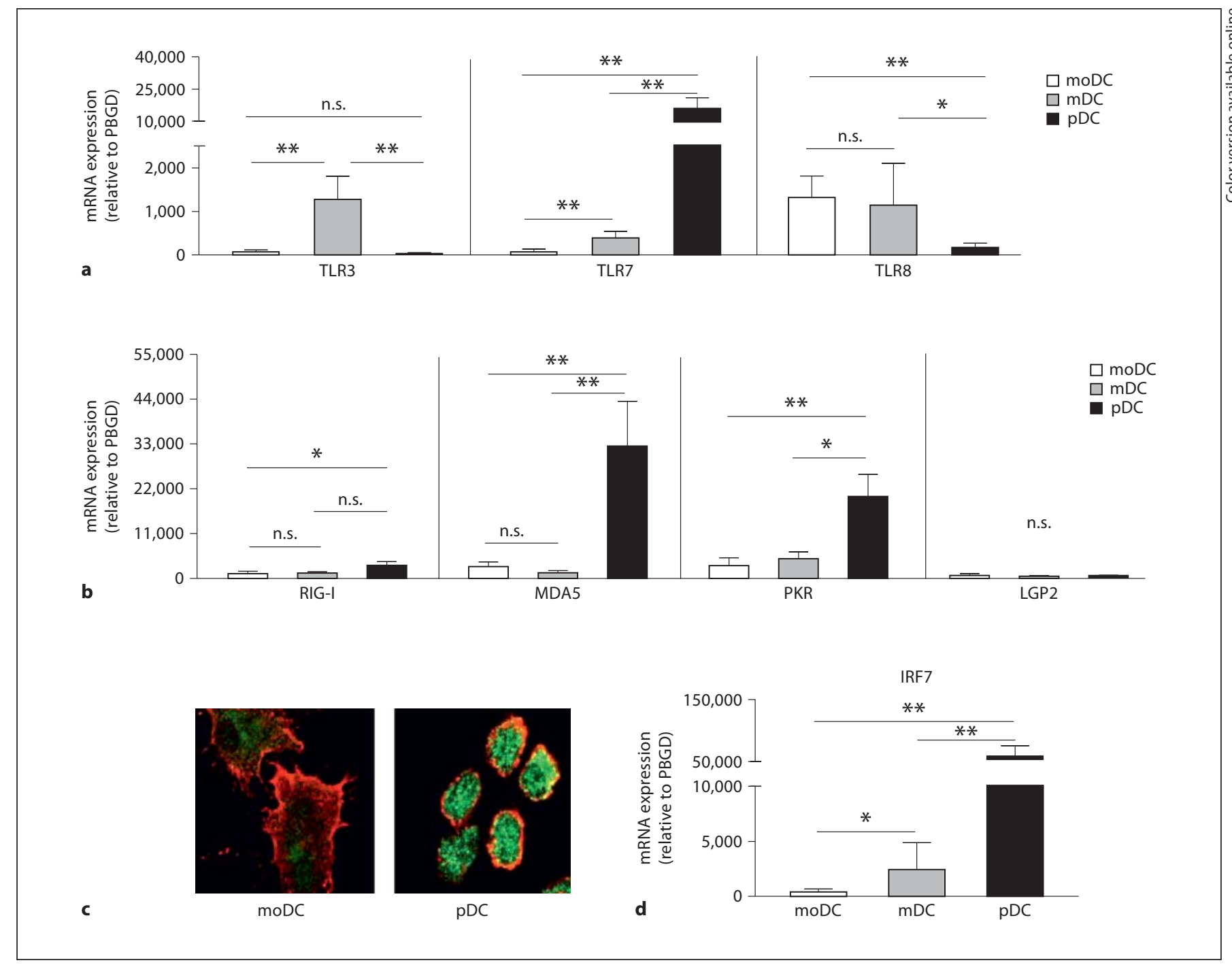

Fig. 2. Human DC subsets express different profiles of viral nucleic acid receptors. a Mean expression (+SD) of TLR3, TLR7 and TLR8 in human moDCs, freshly isolated mDCs and freshly isolated pDCs. RNA was isolated from unstimulated cells and TLR mRNA transcript levels determined using conventional qPCR. Expression levels relative to PBGD. ${ }^{*} \mathrm{p}<0.05 ;{ }^{* *} \mathrm{p}<0.01$. b Expression levels of RIG-I, MDA5, PKR and LGP2 in resting human DC subsets were determined as described for a. Shown is mean expression + SD. ${ }^{*} \mathrm{p}<0.05 ;{ }^{* *} \mathrm{p}<0.01$. c Confocal laser scanning microscopy analysis of MDA5 expression in human moDCs and pDCs. Unstimulated cells were stained using antibodies against
DC-SIGN and MDA5 (for moDCs) or BDCA2 and MDA5 (for pDCs) followed by incubation with Alexa-labeled secondary antibodies. Green indicates MDA5 expression; red indicates DCSIGN or BDCA2 in moDCs and pDCs, respectively. A representative example of 3 independent experiments using different donors is shown. d IRF-7 expression in human DC subsets was determined using qPCR as described for $\mathbf{a}$. Shown is mean expression + SD. ${ }^{*} \mathrm{p}<0.05 ;{ }^{* *} \mathrm{p}<0.01$. Mean quantitative gene expression + $\mathrm{SD}$ from 6 moDCs, $3 \mathrm{pDCs}$ and $3 \mathrm{mDCs}$ is displayed (for $\mathbf{a}, \mathbf{b}$ and d, respectively). Colors refer to the online version only.
mDCs that showed over 20 -fold higher expression compared to moDCs or pDCs. The observed differences in expression of TLR7 were even more dramatic: pDCs showed 40- and over 200-fold higher TLR7 levels as compared to $\mathrm{mDC}$ and moDC (fig. 2a). Thus far, no data is available regarding the expression or function of cytoplasmic RNA sensors in human mDCs or pDCs. We found that all DC subsets expressed mRNA of RIG-I, MDA5, PKR and LGP2 (fig. 2b). Of these genes, LGP2 expression was the lowest in all DC types analyzed, which 
might be related to its proposed function as negative regulator of MDA5 and RIG-I responses [16]. For both myeloid DC subsets only a relatively modest difference was observed in expression levels of RIG-I and MDA5. In contrast, pDCs expressed considerably higher levels of MDA5 compared to RIG-I (fig. 2b). Furthermore, pDCs expressed both RIG-I and PKR at higher levels than moDCs and/or mDCs. The most striking difference was observed for expression of MDA5 mRNA, which was expressed in pDCs at 10-fold and 20-fold higher levels than in moDCs or mDCs, respectively (fig. $2 b$ ). The abundance of MDA5 in pDCs was confirmed at the protein level by confocal microscopy (fig. 2c). The high expression of not only TLR7, but also cytoplasmic RNA sensors and antiviral effector molecules in pDCs provides additional evidence for specialization of DC subsets and the essential contribution of pDCs to antiviral immunity. This was further substantiated by the high constitutive mRNA level of IFN regulatory factor 7 (IRF-7), a key transcription factor in the innate immune response against viruses, which was significantly higher than in moDCs or mDCs (fig. 2d) [24-26].

\section{Plasmacytoid DC Mediated Anti-Viral Immunity in moDCs}

Taking into account the crucial role of $\mathrm{pDC}$ in antiviral immunity, we next investigated whether pDCs could influence antiviral responses in other DC subsets. Therefore, we set out to determine whether activation of pDCs could influence RNA sensor expression in DCs of the myeloid lineage by analyzing RLH levels in moDCs that were exposed to supernatant of pDCs activated with $\mathrm{CpG}$ DNA. No significant direct effect of CpG DNA on moDC was observed (data not shown). Strikingly, pDC supernatant diluted as much as 100 times triggered a 150 - to 300 fold increase in RIG-I, MDA5 and PKR transcript levels in moDC. Similar ISG induction in moDCs was found when using recombinant human IFN- $\alpha 2$ and supernatant from R848-stimulated pDC (fig. 3a; data not shown), suggesting an important role for type I IFNs in this process. Addition of $\mathrm{CpG}$ or R848 to moDC did not induce ISG expression (data not shown; Kramer et al. [27]), excluding the possibility that the observed effects are due to direct PRR stimulation of moDCs. The importance of type I IFNs was confirmed by the use of blocking antiIFN $-\alpha / \beta$ antibodies, which completely prevented the induction of these RNA sensors following stimulation with either pDC supernatant or rIFN- $\alpha 2$ (fig. 3a). The increased mRNA levels of MDA5 in moDCs were confirmed at the protein level by confocal analysis (fig. 3b).
Furthermore, Western blot analysis confirmed increased protein levels of not only MDA5, but also RIG-I and PKR upon IFN stimulation (fig. 3c). Interestingly, MDA5 has been reported to play a crucial role in the responses to the picornavirus encephalomyocarditisvirus (EMCV) in mouse DCs [12] and we recently showed that echoviruses, single-stranded RNA viruses also belonging to the picornavirus family, can efficiently infect human moDCs [18]. To study whether upregulation of viral sensors, including MDA5, was associated with decreased susceptibility for infection, moDCs were exposed to $\mathrm{pDC}$ supernatant and subsequently infected with EV9. EV9 titers rapidly increased in untreated moDCs or moDCs exposed to supernatant of unstimulated pDCs, indicating efficient virus replication. In contrast, treatment with supernatant of CpG-stimulated pDCs completely blocked EV9 replication in human moDCs (fig. 3d).

To study DC cross-talk under more physiological conditions, pDCs were stimulated with CVB3 in the presence or absence of $10 \%$ human serum (HS). As we have shown before, CVB3 is not capable of infecting moDCs, and has no effect on moDC function [18]. However, pDC activation by CVB3 has been reported in the presence of specific antiviral antibodies found in HS [28]. Stimulation of moDC with supernatant of unstimulated pDCs, or CVB3stimulated pDC without HS had little or no effect on expression of RIG-I, MDA5 and PKR mRNA. However, supernatant of $\mathrm{pDC}$ exposed to CVB3 in the presence of $10 \%$ HS caused a profound increase in RNA sensors in moDC (fig. 4a). The observed effects were highly IFN$\alpha / \beta$ dependent, since mRNA induction in the presence of blocking anti-IFN- $\alpha / \beta$ antibodies was completely absent (fig. 4a). pDCs produced up to $40 \mathrm{ng} / \mathrm{ml} \mathrm{IFN}-\alpha$ upon stimulation with CVB3 and human serum (data not shown). This is a substantial increase that even exceeds the induction of IFN- $\alpha$ by CpG (approx. $17 \mathrm{ng} / \mathrm{ml}$; data not shown). To determine if stimulation with virus-activated $\mathrm{pDC}$ supernatant provided functional protection from infection, moDCs were subsequently exposed to EV9. Efficient EV9 replication was observed in moDCs that had been treated with pDC supernatant from mockstimulated pDCs, as indicated by the increase in virus titer. In contrast, moDCs stimulated with supernatant from $\mathrm{pDC}$ that were exposed to CVB3 in the presence of HS were completely protected from EV9 infection, as shown by the dramatic effect on EV9 replication (fig. $4 \mathrm{~b}$ ). Thus, pDC activation can increase the resistance against viral infection in DCs from the myeloid lineage.

In conclusion, we show that endosomal and cytoplasmic viral RNA sensors are widely expressed in moDCs, 


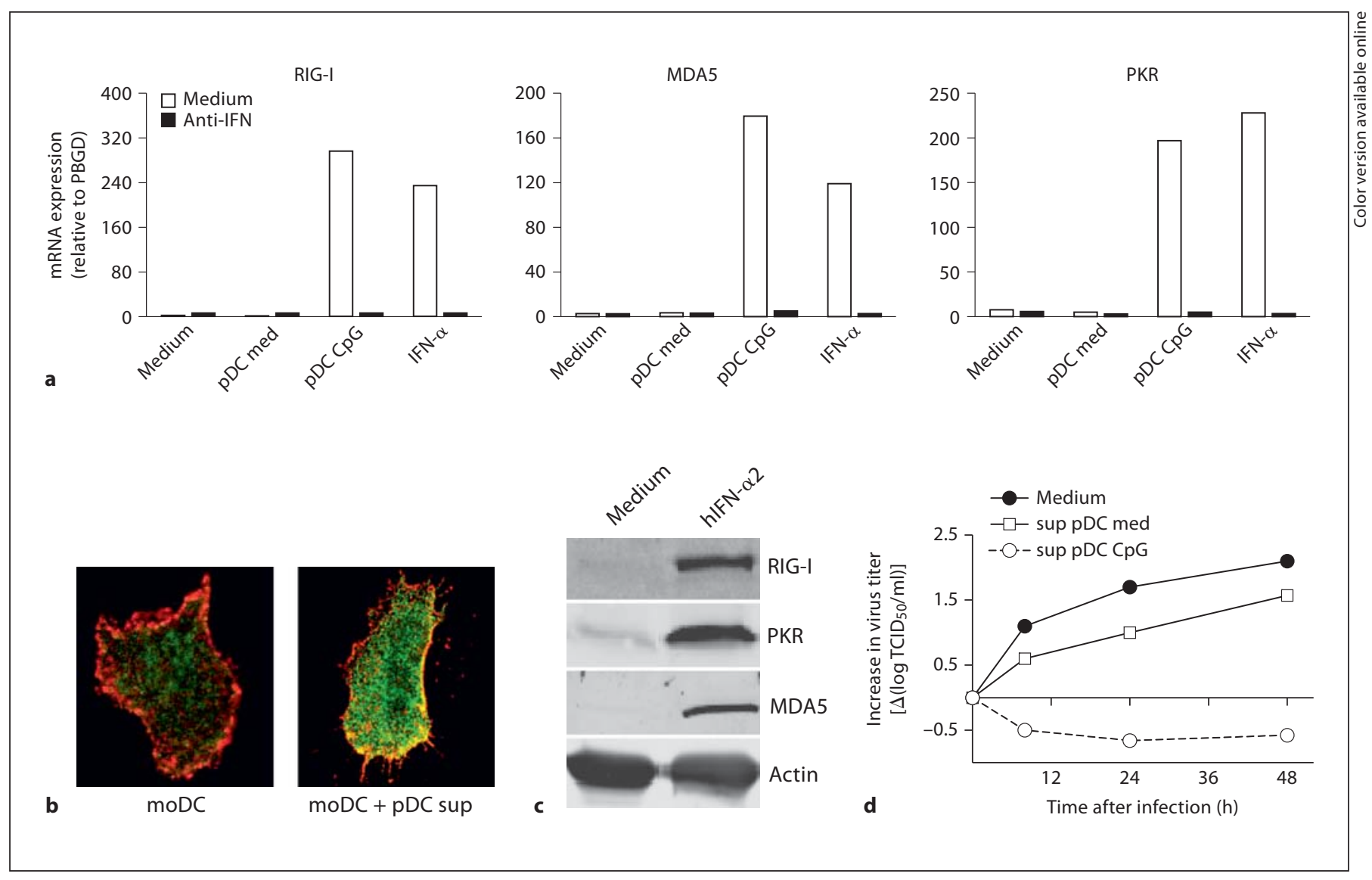

Fig. 3. TLR-stimulated human pDCs induce a state of antiviral resistance in moDCs that inhibits infection with EV9. a Human moDCs were exposed to cell-free supernatants of pDCs (in a 1:100 dilution in RPMI 1640) that had been stimulated with CpG DNA $(5 \mu \mathrm{g} / \mathrm{ml})$ for a period of $24 \mathrm{~h}$. Alternatively, moDCs were stimulated using $100 \mathrm{U} / \mathrm{ml}$ IFN- $\alpha 2$ (Roferon-A; Roche). Total RNA was isolated at the indicated time points following stimulation and mRNA transcript levels determined using qPCR with expression of PBGD as a reference. Expression is given relative to unstimulated cells (medium). b Confocal laser scanning microscopy analysis of MDA5 expression in human moDCs that were left untreated or exposed to supernatant of pDCs activated with CpG DNA as described for $\mathbf{a}$. Cells were stained using antibodies against DCSIGN and MDA5 followed by incubation with Alexa-labeled sec- ondary antibodies. Green indicates MDA5 expression; red indicates expression of DC-SIGN. c Human moDCs were stimulated

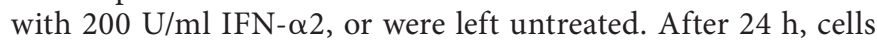
were harvested and protein levels of RIG-I, MDA5, PKR and actin were analyzed using Western blot. Shown is a representative example from 2 independent experiments using different donors. d Human moDCs were exposed to cell-free supernatants of differently stimulated pDCs for a period of $24 \mathrm{~h}$ and subsequently harvested, washed and exposed to EV9 at an MOI of 1 in serumfree medium for $1 \mathrm{~h}$ at $37^{\circ} \mathrm{C}$. After washes to remove unbound virus, cells were plated out and viral titers were determined at several time points after infection. Shown is a representative example of 2 independent experiments using different donors. Colors refer to the online version only. and this expression is increased upon inflammation. Furthermore, under steady-state conditions, these RNA sensors display a differential expression pattern in various human DC subsets, with the most substantial expression found in pDCs. Finally, cross-talk between virus-activated pDCs and moDCs offers protection against viral infection, which underscores the importance of cross-talk between DC subsets in optimal induction of immune responses against invading pathogens.

\section{Discussion}

DCs act as sentinels of the body and are present in virtually all tissues, where they constantly monitor their surroundings for signs of infection. To recognize microorganisms, DCs express a vast array of PRRs. Although a large body of information is available regarding the expression and function of TLRs in DCs, data on expression and function of the different RLH family members in hu- 


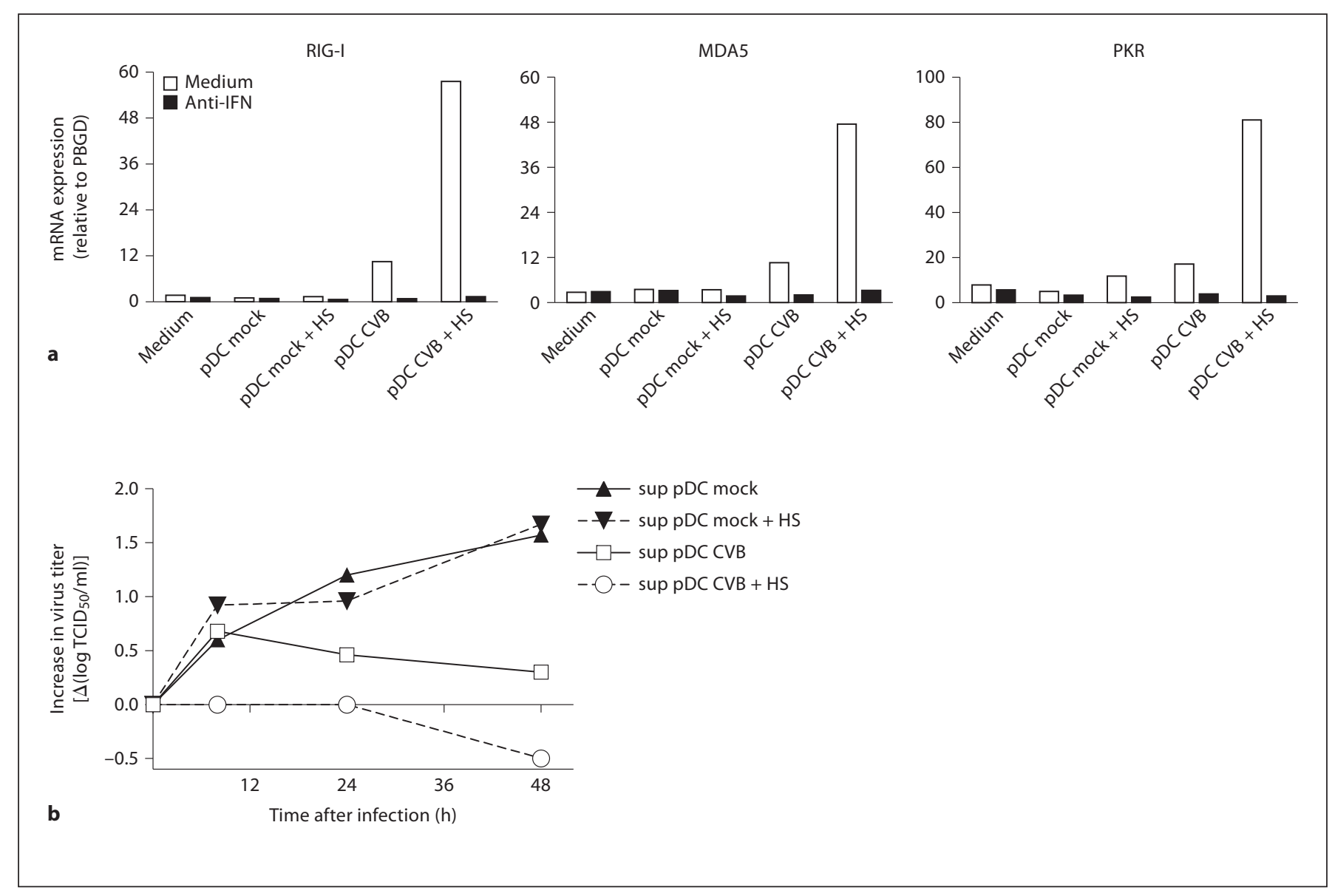

Fig. 4. CVB-stimulated pDCs induce a type I IFN-dependent induction of viral RNA sensors in moDCs and protection from subsequent EV9 infection. a Human moDCs were exposed to cellfree supernatants of pDCs (1:10 dilution in RPMI 1640) that were either mock or CVB stimulated in the presence of absence of $10 \%$ human serum as indicated. Total RNA was isolated following stimulation and mRNA transcript levels were determined using
qPCR with expression of PBGD as a reference. Expression is given relative to unstimulated cells (medium). b moDCs were stimulated with pDC supernatant as described in a for $24 \mathrm{~h}$ and subsequently harvested, infected with EV9 at an MOI of 1. EV9 replication was analyzed as for figure $3 \mathrm{~d}$. Shown is a representative example of 2 independent experiments using different donors. man DCs are virtually absent. We set out to determine the quantitative expression levels of TLRs and RLHs in human DCs at different experimental conditions, focusing on PRRs involved in recognition of viral RNA. Furthermore, we compared steady-state expression levels of these sensors in different DC subsets, and investigated cross-talk between virus-stimulated pDCs and moDCs.

Human moDCs were found to express transcripts for all TLR family members, and most of the C-type lectins analyzed. The mean expression levels of TLRs responding to nucleic acids were considerably lower than those responding to bacterial or fungal pathogens. This could represent a mechanism to prevent unwanted responses to host nucleic acids that have been associated with autoimmune diseases like systemic lupus erythematosus $[29,30]$. On the other hand, expression of PRRs in different cell types can be altered under pro- or anti-inflammatory conditions (this study, Homma et al. [31], Rosenstiel et al. [32] and Fitzner et al. [33]). Under pro-inflammatory conditions (that is LPS/IFN- $\gamma$ ), the expression of TLRs - especially TLR7 and TLR8 - was increased, providing a mechanism to efficiently fight infection upon encounter with pathogens. The upregulation of these molecules was most pronounced at early time points following stimulation, and had declined again after $24 \mathrm{~h}$. The rather swift PRR downregulation most likely functions to prevent un- 
necessary high PRR expression levels once infection has been cleared, thereby avoiding uncontrolled inflammatory responses.

Myeloid DCs and pDCs have been shown to display distinct TLR profiles that enable them to respond to different microbial structures $[4,34]$. We here confirmed these data on a quantitative level and showed that expression of TLR7 in pDCs vastly exceeded that of TLR3 and TLR8. This is in accordance with the findings that pDCs respond strongly to synthetic TLR7, but not TLR8-specific ligands [35], and are unresponsive to the synthetic dsRNA mimic poly(I:C) [4]. In contrast, moDCs expressed much higher levels of TLR8 compared to TLR3 and TLR7. The highly divergent expression profile of TLR7 and TLR8 in human pDCs and myeloid DC subsets likely reflects their ability to induce specific responses following recognition of different virus structures.

Virtually no data is currently available regarding RIGI and MDA5 in human DC subsets. A recent mouse study has suggested that RIG-I is crucial for the antiviral response of myeloid DCs following exposure to the RNA virus Newcastle disease virus. In contrast, in pDCs RIGI was shown to be dispensable and the antiviral responses against Newcastle disease virus in mice were dependent on the TLR signaling pathway [16]. Interestingly, our finding that RIG-I mRNA levels in human pDCs were significantly higher than the levels found in moDCs suggests that in the human setting, RIG-I could play a pivotal role in the response of pDCs to RNA viruses. Even more pronounced were the expression levels of MDA5 in human $\mathrm{pDCs}$ that were remarkably higher than those found in moDCs or mDCs. MDA5 has recently been shown to mediate type I IFN responses following exposure of mouse myeloid DCs to poly(I:C) and the picornavirus EMCV [12]. Whether the prominent expression of MDA5 signifies involvement of this molecule in the response of pDCs to picornaviruses remains to be determined. It could be envisioned that the high constitutive levels of TLR7, RLHs and PKR in pDCs might not only mediate the robust type I IFN production of pDCs in response to viral pathogens but could also protect these cells from becoming infected themselves.

The cytoplasmic localization of RIG-I and MDA5 suggests that they play a dominant role in case of infection of DCs themselves. However, it could be envisioned that viral replication intermediates can come into contact with these molecules upon phagocytosis of infected cells, as it has been shown that antigen delivery from the endosomal compartment to the cytosol takes places in DCs, for instance to facilitate cross-presentation [36-38]. Howev- er, whether these pathways can efficiently contribute to the RIG-I- or MDA5-mediated antiviral responses remains to be determined.

Finally, we revealed a novel effect of cross-talk between pDCs and DCs of myeloid origin. It has been described that cross-talk between pDCs and mDCs can influence cytokine production, DC maturation and antigen presentation [3, 39-41]. In this study, we showed that cross-talk can induce rapid upregulation of RNA sensors in moDCs and protection against infection with picornaviruses. This protection depended on type I IFNs, cytokines with well-known anti-viral activity that signal through the ubiquitously expressed type I IFN receptor. Myeloid DCs are known to respond to IFN- $\alpha$ [40, 42, 43], but pDC-derived type I IFNs are known to affect many other cell types when present in their vicinity. For example, it has been shown that type I IFNs can influence expression of RLHs in keratinocytes and macrophages [4446]. Importantly, our study revealed that protection of moDC by pDC-derived type I IFNs not only occured upon $\mathrm{pDC}$ stimulation using synthetic TLR ligands, but also following a more physiological exposure to CVB. The finding that $\mathrm{pDC}$-mediated protection largely depended on the presence of human serum during virus exposure is in line with earlier result by Wang et al. [28]. Most likely, virus particles coated with anti-viral antibodies are being taken up via an FcR-mediated process, which ultimately leads to activation of the pDC through TLRs and possibly other PRRs [28]. This antibody-mediated process depends on the presence of specific anti-viral antibodies induced following activation of B cells. Hence, it is likely to be more efficient upon secondary encounter with the same virus, when antibodies are already present and production is enhanced. Thus, pDCs could preserve the function and viability of myeloid DCs in case of infection with DC-tropic viruses. This will ensure antigen presentation to both CTL and Th cells, thereby enhancing the antiviral immune response. On the other hand, pDCs might mediate unbalanced responses by myeloid DCs or T cells [47], possibly contributing to immune-mediated diseases associated with CVB infections, such as cardiomyopathy and type 1 diabetes $[48,49]$.

In conclusion, we demonstrate for the first time that human moDCs express high mRNA and protein levels of RLHs and PKR, which show a transient increase following exposure to pro-inflammatory stimuli. Expression profiling of PRRs involved in the antiviral response in different human DC subsets demonstrated that pDCs express significantly higher levels of RLHs, PKR and IRF-7 compared to myeloid DCs. The profound expression of 
these antiviral genes in human pDCs is in line with their important function in antiviral immunity, and might also explain how these cells survive and function in an environment with high virus burden. Finally, cross-talk between virus-stimulated pDCs and moDCs enhanced the expression of RIG-I, MDA5 and PKR in moDCs and created a state of antiviral resistance that protected these cells against viral infection. Further investigation into the novel findings presented here regarding the effect of human DC cross-talk on RLH expression and antiviral resistance will contribute significantly to our understanding of DC-pathogen interaction.

\section{Acknowledgments}

The authors would like to thank Daniel Benitez-Ribas (Department of Tumor Immunology, Nijmegen Centre for Molecular Life Sciences, Radboud University Medical Centre, Nijmegen, The Netherlands) for help with pDC experiments, Paul Fisher (Departments of Pathology, Neurosurgery and Urology, Columbia University, College of Physicians and Surgeons, New York, N.Y., USA) for providing us with anti-MDA5 antibody and Harrie van der Avoort (National Institute for Public Health and the Environment, RIVM, Bilthoven, The Netherlands) for providing neutralizing anti-CVB3 antibody. This work was supported by grants from the Netherlands Diabetes Foundation (DFN 2001.00.047) to G.J.A., the Netherlands Organization for Scientific Research (NWO-VIDI-917.46.305) to F.J.M.v.K. and (NWO912-02-034) to G.J.A. and the Juvenile Diabetes Research Foundation (grant No. 24-2008-949) to G.J.A. and F.J.M.v.K.

\section{References}

1 Banchereau J, Steinman RM: Dendritic cells and the control of immunity. Nature 1998; 392:245-252.

-2 Agrawal S, Agrawal A, Doughty B, Gerwitz A, Blenis J, Van Dyke T, Pulendran B: Cutting edge: different toll-like receptor agonists instruct dendritic cells to induce distinct th responses via differential modulation of extracellular signal-regulated kinase-mitogen-activated protein kinase and c-fos. J Immunol 2003;171:4984-4989.

-3 Ito T, Kanzler H, Duramad O, Cao W, Liu YJ: Specialization, kinetics, and repertoire of type 1 interferon responses by human plasmacytoid predendritic cells. Blood 2006;107: 2423-2431.

4 Kadowaki N, Ho S, Antonenko S, Malefyt RW, Kastelein RA, Bazan F, Liu YJ: Subsets of human dendritic cell precursors express different toll-like receptors and respond to different microbial antigens. J Exp Med 2001;194:863-869.

5 Ito T, Amakawa R, Kaisho T, Hemmi H, Tajima K, Uehira K, Ozaki Y, Tomizawa $\mathrm{H}$, Akira S, Fukuhara S: Interferon- $\alpha$ and interleukin-12 are induced differentially by tolllike receptor 7 ligands in human blood dendritic cell subsets. J Exp Med 2002;195: 1507-1512.

-6 Sallusto F, Lanzavecchia A: Efficient presentation of soluble antigen by cultured human dendritic cells is maintained by granulocyte/ macrophage colony-stimulating factor plus interleukin 4 and downregulated by tumor necrosis factor alpha. J Exp Med 1994;179: 1109-1118.

7 Osugi Y, Vuckovic S, Hart DN: Myeloid blood CD11c(+) dendritic cells and monocyte-derived dendritic cells differ in their ability to stimulate $\mathrm{T}$ lymphocytes. Blood 2002;100:2858-2866.
8 Karlsson A, Nygren E, Karlsson J, Nordstrom I, Dahlgren C, Eriksson K: Ability of monocyte-derived dendritic cells to secrete oxygen radicals in response to formyl peptide receptor family agonists compared to that of myeloid and plasmacytoid dendritic cells. Clin Vaccine Immunol 2007;14:328330.

-9 Medzhitov R: Recognition of microorganisms and activation of the immune response. Nature 2007;449:819-826.

10 Macagno A, Napolitani G, Lanzavecchia A, Sallusto F: Duration, combination and timing: The signal integration model of dendritic cell activation. Trends Immunol 2007;28: 227-233.

11 Kato H, Takeuchi O, Sato S, Yoneyama M, Yamamoto M, Matsui K, Uematsu S, Jung A, Kawai T, Ishii KJ, Yamaguchi O, Otsu K, Tsujimura T, Koh CS, Reis e Sousa C, Matsuura Y, Fujita T, Akira S: Differential roles of MDA5 and RIG-I helicases in the recognition of RNA viruses. Nature 2006;441:101105.

12 Gitlin L, Barchet W, Gilfillan S, Cella M, Beutler B, Flavell RA, Diamond MS, Colonna M: Essential role of MDA-5 in type I IFN responses to polyriboinosinic: polyribocytidylic acid and encephalomyocarditis picornavirus. Proc Natl Acad Sci USA 2006;103: 8459-8464.

13 Takahasi K, Yoneyama M, Nishihori T, Hirai R, Kumeta H, Narita R, Gale M, Jr., Inagaki F, Fujita T: Nonself RNA-sensing mechanism of RIG-I helicase and activation of antiviral immune responses. Mol Cell 2008;29: 428-440.
14 Schlee M, Roth A, Hornung V, Hagmann CA, Wimmenauer V, Barchet W, Coch C, Janke M, Mihailovic A, Wardle G, Juranek S, Kato H, Kawai T, Poeck H, Fitzgerald KA, Takeuchi O, Akira S, Tuschl T, Latz E, Ludwig J, Hartmann G: Recognition of $5^{\prime}$ triphosphate by RIG-I helicase requires short blunt double-stranded RNA as contained in panhandle of negative-strand virus. Immunity 2009

15 Takeuchi O, Akira S:MDA5/RIG-I and virus recognition. Curr Opin Immunol 2008;20: $17-22$.

16 Yoneyama M, Kikuchi M, Matsumoto K, Imaizumi T, Miyagishi M, Taira K, Foy E, Loo YM, Gale M Jr, Akira S, Yonehara S, Kato A, Fujita T: Shared and unique functions of the DExD/H-box helicases RIG-I, MDA5, and LGP2 in antiviral innate immunity. J Immunol 2005;175:2851-2858.

17 Clemens MJ, Elia A: The double-stranded RNA-dependent protein kinase PKR: structure and function. J Interferon Cytokine Res 1997;17:503-524.

18 Kramer M, Schulte BM, Toonen LW, de Bruijni MA, Galama JM, Adema GJ, van Kuppeveld FJ: Echovirus infection causes rapid loss-of-function and cell death in human dendritic cells. Cell Microbiol 2007;9: 1507-1518

19 Wang X, Seed B: A PCR primer bank for quantitative gene expression analysis. Nucleic Acids Res 2003;31:e154.

-20 Lin L, Su Z, Lebedeva IV, Gupta P, Boukerche H, Rai T, Barber GN, Dent P, Sarkar D, Fisher PB: Activation of RAS/RAF protects cells from melanoma differentiation-associated gene-5-induced apoptosis. Cell Death Differ 2006;13:1982-1993. 
21 R Development Core Team: R: A Language and Environment for Statistical Computing. Vienna, R Foundation for Statistical Computing, 2005.

-22 Geijtenbeek TB, Kwon DS, Torensma R, van Vliet SJ, van Duijnhoven GC, Middel J, Cornelissen IL, Nottet HS, KewalRamani VN, Littman DR, Figdor CG, van Kooyk Y: DCSIGN, a dendritic cell-specific HIV-1-binding protein that enhances trans-infection of T cells. Cell 2000;100:587-597.

-23 Cambi A, de Lange F, van Maarseveen NM, Nijhuis M, Joosten B, van Dijk EM, de Bakker BI, Fransen JA, Bovee-Geurts PH, van Leeuwen FN, Van Hulst NF, Figdor CG: Microdomains of the c-type lectin DC-SIGN are portals for virus entry into dendritic cells. J Cell Biol 2004;164:145-155.

$\checkmark 24$ Coccia EM, Severa M, Giacomini E, Monneron D, Remoli ME, Julkunen I, Cella M, Lande R, Uze G: Viral infection and Toll-like receptor agonists induce a differential expression of type I and $\lambda$ interferons in human plasmacytoid and monocyte-derived dendritic cells. Eur J Immunol 2004;34:796-805.

-25 Izaguirre A, Barnes BJ, Amrute S, Yeow WS, Megjugorac N, Dai J, Feng D, Chung E, Pitha PM, Fitzgerald-Bocarsly P: Comparative analysis of IRF and IFN- $\alpha$ expression in human plasmacytoid and monocyte-derived dendritic cells. J Leukoc Biol 2003;74:11251138 .

26 Kerkmann M, Rothenfusser S, Hornung V, Towarowski A, Wagner M, Sarris A, Giese T, Endres S, Hartmann G: Activation with CPG-a and CPG-b oligonucleotides reveals two distinct regulatory pathways of type I IFN synthesis in human plasmacytoid dendritic cells. J Immunol 2003;170:4465-4474.

-27 Kramer M, Schulte BM, Toonen LW, Barral PM, Fisher PB, Lanke KH, Galama JM, van Kuppeveld FJ, Adema GJ: Phagocytosis of picornavirus-infected cells induces an RNAdependent antiviral state in human dendritic cells. J Virol 2008;82:2930-2937.

-28 Wang JP, Asher DR, Chan M, Kurt-Jones EA, Finberg RW: Cutting edge: antibody-mediated TLR7-dependent recognition of viral RNA. J Immunol 2007;178:3363-3367.

29 Means TK, Luster AD: Toll-like receptor activation in the pathogenesis of systemic lupus erythematosus. Ann NY Acad Sci 2005; 1062:242-251.
30 Deane JA, Pisitkun P, Barrett RS, Feigenbaum L, Town T, Ward JM, Flavell RA, Bolland S: Control of toll-like receptor 7 expression is essential to restrict autoimmunity and dendritic cell proliferation. Immunity 2007 27:801-810.

31 Homma T, Kato A, Hashimoto N, Batchelor J, Yoshikawa M, Imai S, Wakiguchi H, Saito H, Matsumoto K: Corticosteroid and cytokines synergistically enhance toll-like receptor 2 expression in respiratory epithelial cells. Am J Respir Cell Mol Biol 2004;31:463469.

32 Rosenstiel P, Fantini M, Brautigam K, Kuhbacher T, Waetzig GH, Seegert D, Schreiber S: TNF- $\alpha$ and IFN- $\gamma$ regulate the expression of the NOD2 (CARD15) gene in human intestinal epithelial cells. Gastroenterology 2003;124:1001-1009.

33 Fitzner N, Clauberg S, Essmann F, Liebmann J, Kolb-Bachofen V: Human skin endothelial cells can express all 10 TLR genes and respond to respective ligands. Clin Vaccine Immunol 2008;15:138-146.

-34 Jarrossay D, Napolitani G, Colonna M, Sallusto F, Lanzavecchia A: Specialization and complementarity in microbial molecule recognition by human myeloid and plasmacytoid dendritic cells. Eur J Immunol 2001;31: 3388-3393.

-35 Gorden KB, Gorski KS, Gibson SJ, Kedl RM, Kieper WC, Qiu X, Tomai MA, Alkan SS, Vasilakos JP: Synthetic TLR agonists reveal functional differences between human TLR7 and TLR8. J Immunol 2005;174:12591268.

- 36 Rodriguez A, Regnault A, Kleijmeer M, Ricciardi-Castagnoli P, Amigorena S: Selective transport of internalized antigens to the $\mathrm{cy}$ tosol for MHC class I presentation in dendritic cells. Nat Cell Biol 1999;1:362-368.

37 Hotta C, Fujimaki H, Yoshinari M, Nakazawa M, Minami M: The delivery of an antigen from the endocytic compartment into the cytosol for cross-presentation is restricted to early immature dendritic cells. Immunology 2006;117:97-107.

38 Ramirez MC, Sigal LJ: Macrophages and dendritic cells use the cytosolic pathway to rapidly cross-present antigen from live, vaccinia-infected cells. J Immunol 2002;169: 6733-6742.

-39 Kuwajima S, Sato T, Ishida K, Tada H, Tezuka $\mathrm{H}$, Ohteki T: Interleukin 15-dependent crosstalk between conventional and plasmacytoid dendritic cells is essential for CPGinduced immune activation. Nat Immunol 2006;7:740-746.
40 Piccioli D, Sammicheli C, Tavarini S, Nuti S, Frigimelica E, Manetti AG, Nuccitelli A, Aprea S, Valentini S, Borgogni E, Wack A, Valiante NM: Human plasmacytoid dendritic cells are unresponsive to bacterial stimulation and require a novel type of cooperation with myeloid dendritic cells for maturation. Blood 2009;113:4232-4239.

- 41 Lou Y, Liu C, Kim GJ, Liu YJ, Hwu P, Wang G: Plasmacytoid dendritic cells synergize with myeloid dendritic cells in the induction of antigen-specific antitumor immune responses. J Immunol 2007;178:1534-1541.

42 Kanto T, Hayashi N: Innate immunity in hepatitis $C$ virus infection: interplay among dendritic cells, natural killer cells and natural killer T cells. Hepatol Res 2007;37(suppl 3):S319-S326.

43 Kanto T: Virus associated innate immunity in liver. Front Biosci 2008;13:6183-6192.

44 Kang DC, Gopalkrishnan RV, Wu Q, Jankowsky E, Pyle AM, Fisher PB: MDA-5: an interferon-inducible putative RNA helicase with double-stranded RNA-dependent ATPase activity and melanoma growth-suppressive properties. Proc Natl Acad Sci USA 2002;99:637-642.

-45 Prens EP, Kant M, van Dijk G, van der Wel LI, Mourits S, van der Fits L: IFN- $\alpha$ enhances poly-IC responses in human keratinocytes by inducing expression of cytosolic innate RNA receptors: Relevance for psoriasis. J Invest Dermatol 2008;128:932-938.

46 Siren J, Pirhonen J, Julkunen I, Matikainen $S:$ IFN- $\alpha$ regulates TLR-dependent gene expression of IFN- $\alpha$, IFN- $\beta$, IL-28, and IL-29. J Immunol 2005;174:1932-1937.

47 Benitez-Ribas D, Adema GJ, Winkels G, Klasen IS, Punt CJ, Figdor CG, de Vries IJ: Plasmacytoid dendritic cells of melanoma patients present exogenous proteins to $\mathrm{CD} 4+$ T cells after Fc gamma RII-mediated uptake. J Exp Med 2006;203:1629-1635.

48 Hyoty H, Taylor KW: The role of viruses in human diabetes. Diabetologia 2002;45: 1353-1361.

-49 Schwimmbeck PL, Bigalke B, Schulze K, Pauschinger M, Kuhl U, Schultheiss HP: The humoral immune response in viral heart disease: characterization and pathophysiological significance of antibodies. Med Microbiol Immunol 2004;193:115-119. 